\title{
Type B2 thymoma invading the superior vena cava and right atrium and with lung metastases: $A$ young case report
}

\section{Jia-Ju Lu}

The First Hospital of Lanzhou University

Hong-Lin Zhao

The First Hospital of Lanzhou University

\section{Yun-Dan Deng}

The First Hospital of Lanzhou University

Jin-Rong Ni

The First Hospital of Lanzhou University

Bing Song ( $\nabla$ songbingldyyxwk@sina.com )

The First Hospital of Lanzhou University

\section{Case Report}

Keywords: Thymoma, Superior vena cava, Right atrium, Lung metastases, Age, Chemotherapy

Posted Date: February 25th, 2022

DOI: https://doi.org/10.21203/rs.3.rs-1374946/v1

License: (c) (1) This work is licensed under a Creative Commons Attribution 4.0 International License. Read Full License 


\section{Abstract}

\section{BACKGROUND}

Thymoma is a relatively rare type of tumor. It is more common in middle-aged patients than in children and young adults. We report a 26-year-old patient with advanced giant thymomas. The patient's tumor have invaded the upper venous vein. There was also a large tumor in the right atrium. The patient had lung metastases.

\section{CASE SUMMARY}

We report a patient with type B2 thymoma. His image studies showed a mediastinal space-occupying lesion that penetrated through the superior vena cava and extended into the right atrium, with metastases in the right lung and enlarged lymph nodes in the neck, mediastinum, and bilateral axillary areas. Biopsy reports suggested type B2 thymoma. Multidisciplinary consultation concluded with a diagnosis of stage IVB thymoma. This patient began a chemotherapy regimen with cisplatin, doxorubicin, and cyclophosphamide (CAP). Subsequent management decisions were made based on the treatment responses.

\section{CONCLUSION}

At present, thymoma treatment is mainly surgical. However, for patients with advanced thymoma, surgical treatment may risk the patient's survival. For advanced thymoma patients, we recommend chemotherapy (CAP), which may provide a new treatment option for patients with advanced thymoma.

\section{Background}

Thymic epithelial tumors, including thymoma and thymic carcinoma, originate from the thymus and are the most common primary mediastinal tumors ${ }^{[1]}$. Thymoma is a relatively rare type of tumor with an incidence of approximately 2.73 per 1 million population in China ${ }^{[2]}$. It occurs more frequently in adults between 40 and 70 years old and has been occasionally reported in young adults and children ${ }^{[3]}$. Thymomas could be invasive, but its intracardiac growth was extremely rare. In the present study, we report a rare case of a young patient with thymoma invading the superior vena cava and the right atrium, as well as with distant metastases.

\section{Case Presentation}

A 26-year-old male patient presented to our department with chest tightness, shortness of breath, and facial edema for more than one year. The echocardiogram showed a $40 \times 36 \mathrm{~mm}$ first-grade echogenic mass in the right atrium, with clear boundaries and regular shape. The mass had uniform internal echogenicity and great mobility. It was embedded in the tricuspid valve orifice during diastole and fell into the right atrium during systole. In addition, color Doppler showed relative stenosis of the tricuspid valve. 
There was also a hypoechogenic mass in the superior vena cava (Figure 1). Considering that the masses in the right atrium and the superior vena cava were of the same origin, we performed an enhanced computerized tomography (CT) scan. The results showed a large lobulated mediastinal soft tissue mass with small internal vascular structures. The mass penetrated through the superior vena cava and extended into the right atrium. There were enlarged lymph nodes in the neck, mediastinum, and bilateral axillary areas. Both sides of the lungs had multiple solid nodules, and the largest one measured $12 \times 10$ $\mathrm{mm}$ in the lateral basal segment of the lower lobe of the right lung, which was considered a pulmonary metastatic lesion (Figure 2). Cardiac magnetic resonance imaging (MRI) scan reported similar findings (Figure 3).

The patient underwent percutaneous puncture biopsy. The pathological reports showed fibrous septations in certain areas of the specimen with local infiltration of abundant dense cells. Most cells were scattered proliferating lymphoid cells with some proliferating oval cells and epithelioid cells. Immunohistochemical analysis indicated CK19 (some epithelial cells +), CD3 (abundant T lymphocytes +), CD20 (focal +), CD5 (abundant T lymphocytes +), CD1a (some immature T lymphocytes +), CD117 (-), EMA (focal +), vimentin ( $3+$ ), p53 (epithelial 5\%, variable nuclear staining +), ki67 (epithelial 5\%). After combination of morphology and immunohistochemistry results, the final diagnosis was type B2 thymoma (Figure 4). The tumor stage was considered Masaoka-Koga IVB/TNM stage IVB $\left(T_{4} N_{2} M_{1 b}\right)$ based on the pulmonary and distant lymph node metastases. During his hospital stay, this patient did not show any clinical signs of myasthenia gravis. After multidisciplinary discussion, surgical resection was determined not to be option due to the large size of the tumor. The patient was started on a chemotherapy regimen with cyclophosphamide, adriamycin, and platinum (CAP). If the tumor had regressed to the point of resectabilty after the chemotherapy, surgical treatment would be considered again.

\section{Discussion}

Thymoma is an indolent tumor and has an insidious onset with no obvious clinical symptoms in about $50 \%$ of patients ${ }^{[4]}$. It is currently considered as a malignant tumor due to its invasive nature and a high chance of recurrence ${ }^{[5]}$. Thymoma can present with local infiltrations, but it rarely invades the myocardium or intracardiac structures. This enhanced CT and cardiac MRI scans showed that the tumor had penetrated through the superior vena cava and extended into the right atrium. In addition, distant enlarged lymph nodes and multiple solid nodules were found in both lungs. These were considered tumor metastases. Although thymoma usually grows slowly, previous literature has reported distant metastases in the cervical lymph nodes, lungs, and liver ${ }^{[6]}$. There is still debate about the best treatment options for thymoma patients with lung metastases.

Surgical resection is the mainstay treatment for patients with thymoma and is the treatment of choice for patients with Masaoka-Koga stage I-III thymoma and some patients with stage IVA thymoma. The completeness of tumor resection is an independent prognostic factor for thymoma. Thymoma patients 
who undergo R0 resection have shown better prognosis than other patients ${ }^{[7]}$. The prognosis of thymoma patients has also been found to be closely related to the Masaoka-Koga stage. Patients with MasaokaKoga stage IVB thymoma and local invasion, dissemination, recurrence, or distant metastases have a low overall survival rate and a 5 -year survival rate of $11-50 \%[8]$. Platinum-based chemotherapy is recommended for patients with stage IVB thymoma because surgery cannot completely remove the tumor. However, it remains unclear whether the chemotherapy can prolong the survival in these patients.

We searched PubMed, Web of Science, EMBASE, and MEDLINE for related case reports in the 2011-2021 (Table 1) and found that the patients in these case reports were $39-76$ years old ${ }^{[9-18]}$. Weis et al. ${ }^{[19]}$ reported that the age was an independent risk factor for the prognosis. Older patients commonly have poor prognoses and younger patients more frequently had tumor recurrence and metastasis. Our patient was only 26 years old and had wide and distant tumor metastases, which meant he was not a candidate for surgical resection. We started him with CAP chemotherapy (cisplatin $50 \mathrm{mg} / \mathrm{m}^{2} \mathrm{iv}$, doxorubicin 50 $\mathrm{mg} / \mathrm{m}^{2} \mathrm{iv}$, and cyclophosphamide $500 \mathrm{mg} / \mathrm{m}^{2}$ iv, once every 3 weeks), which is the first recommended regimen in the National Comprehensive Cancer Network (NCCN) thymoma guidelines. The survival benefit of the CAP regimen for Masaoka-Koga stage IVB thymoma patients remains to be determined and will require by additional clinical data.

Table 1

Case reports in 2011-2021

\begin{tabular}{|lllll|}
\hline Reference & Year of publication & Age of patient (years) & Sex & Thymoma subtype \\
\hline Takebayashi et al. ${ }^{[9]}$ & 2021 & 71 & Female & Type AB \\
\hline Singh et al. ${ }^{[10]}$ & 2020 & 76 & Male & Type A \\
\hline Shen et al. ${ }^{[1]]}$ & 2020 & 63 & Female & Type B1 and B2 \\
\hline Chen et al. ${ }^{[12]}$ & 2020 & 39 & Female & Type B2 and B3 \\
\hline Yang et al. ${ }^{[13]}$ & 2019 & 50 & Female & Type B3 \\
\hline Lim and Shen ${ }^{[14]}$ & 2017 & 53 & Male & Type B2 \\
\hline Afzal et al. ${ }^{[15]}$ & 2016 & 74 & Female & Type B1 \\
\hline De Giacomo et al. ${ }^{[16]}$ & 2014 & 41 & Male & Type B3 \\
\hline Dong et al.. ${ }^{[17]}$ & 2014 & 75 & Male & Type B3 \\
\hline De Giacomo et al. ${ }^{[16]}$ & 2013 & 44 & Female & Type B3 \\
\hline
\end{tabular}

\section{Conclusion}


In conclusion, we describe a relatively rare young case about thymoma. Due to the giant tumor, wide and distant tumor metastasis, we intend to chemotherapy. Chemotherapy treatment of advanced thymoma has less clinical data. This report may provide a clinical data support for advanced thymoma in chemotherapy treatment.

\section{Abbreviations}

\begin{tabular}{|ll|}
\hline CT & Computerized Tomography \\
\hline MRI & Magnetic Resonance Imaging \\
\hline CAP & cyclophosphamide, adriamycin, platinum \\
\hline
\end{tabular}

\section{Declarations}

ETHICS APPROVAL AND CONSENT TO PARTICIPATE: Not applicable

DATA/INFORMATIONG FOR CONSENT FOR PUBLICATION: The authors declare that the written informed consent was obtained from the patient for publication of this case report and any accompanying images. A copy of the written consent is available for review by the Editor of this journal.

AVAILABILITY OF DATA AND MATERIALS: The datasets used and/or analysed during the current study are available from the corresponding author on reasonable request.

COMPETING INTERESTS: The authors declare that they have no competing interests.

FUNDING: Not applicable.

AUTHOR CONTRIBUTIONS: Jia-Ju Lu, Yun-Dan Deng and Hong-Lin Zhao were the patients` Cardiac surgeon; Jia-Ju Lu reviewed the literature and contributed to manuscript drafting; Yun-Dan Deng and Hong-Lin Zhao analyzed the imaging finding and proposed treatment recommendations. Jin-Rong Ni was sonographer and interpreted the echocardiogram; Bing Song were responsible for the revision of the manuscript for important intellectual content; all authors read and approved the final manuscript.

ACKNOWLEDGEMENTS: We appreciated Xiu-Chao He for image data post-processing the manuscript.

\section{References}

1. He JW, Wei Q. 2019 China cancer registry annual report. Beijing: People's Health Publishing House, 2020: 216-217

2. Travis WD, Brambilla E, Burke AP, Marx A, Nicholson AG. WHO classification of tumours of the lung, pleura, thymus and heart. 4th ed. Geneva: World Health Organization, 2015 
3. Yamada Y, Yoshino I, Nakajima J, Miyoshi S, Ohnuki T, Suzuki M, Nagayasu T, Iwasaki A, Okumura M. Surgical outcomes of patients with stage III thymoma in the Japanese nationwide database. Ann Thorac Surg 2015; 100: 961-967 [PMID: 26163354 DOI: 10.1016/j.athoracsur.2015.04.059]

4. Schmidt-Wolf IGH, Rockstroh JK, Schüller H, Hirner A, Grohe C, Müller-Hermelink HK, Huhn D. Malignant thymoma: Current status of classification and multimodality treatment. Ann Hematol 2003; 82: 69-76 [PMID: 12601482 DOI: 10.1007/s00277-002-0597-6]

5. De Tterbeck FC, Zeeshan A. Thymoma: Current diagnosis and treatment. Chin Med J (Engl) 2013; 126: 2186-2191 [PMID: 23769581 DOI: 10.3760/cma.j.issn.0366-6999.20130177]

6. Hirono M, Nonaka M, Himuro N, Tomita Y, Kataoka D, Kadokura M. Two cases of thymoma with pulmonary metastasis: A case report. World J Surg Oncol 2014; 12: 114 [PMID: 24758419 PMCID: PMC4022537 DOI: 10.1186/1477-7819-12-114]

7. Zhao Y, Shi J, Fan L, Hu D, Yang J, Zhao H. Surgical treatment of thymoma: An 11-year experience with 761 patients. Eur J Cardiothorac Surg 2016; 49: 1144-1149 [PMID: 26324679 DOI: 10.1093/ejcts/ezv288]

8. Kunitoh H, Tamura T, Shibata T, Nakagawa K, Takeda K, Nishiwaki Y, Osaki Y, Noda K, Yokoyama A, Saijo N, Jcog Lung Cancer Study Group TJ. A phase-II trial of dose-dense chemotherapy in patients with disseminated thymoma: Report of a Japan Clinical Oncology Group trial (JCOG 9605). Br J Cancer 2009; 101: 1549-1554 [PMID: 19809436 PMCID: PMC4022537 DOI: 10.1038/sj.bjc.6605347]

9. Takebayashi S, Yoshikawa Y, Morita M, Nagashima R, Nakazono Y, Miyamoto S. Myasthenic crisis appearing after resection of intracardiac ectopic thymoma with superior vena cava syndrome. Surg Case Rep 2021; 7: 144 [PMID: 34131826 PMCID: PMC7542122 DOI: 10.1186/s40792-021-01233-4]

10. Singh N, Nand P, Alison P. Complex redo surgery to treat a large thymoma invading the right atrium. $J$ Card Surg 2020; 35: 1368-1370 [PMID: 32333440 DOI: 10.1111/jocs.14571]

11. Shen W, Cao Y, Wang X, Zhang P, Zhou Q. Invasive thymoma with intravascular growth into the great veins and right atrium: A case report. Thorac Cancer 2020; 11: 1326-1329 [PMID: 32180340 PMCID: PMC7180703 DOI: 10.1111/1759-7714.13242]

12. Chen Z-X, Xiang X-R, Liu R-S, Feng Y, Nan J, Xue J-M, Lei J-Q, Guo S-L. Invasive thymoma extending into the superior vena cava and right atrium: The value of multimodal cardiac magnetic resonance. $J$ Cardiol Cases 2020; 22: 283-285 [PMID: 33304422 PMCID: PMC7718505 DOI:

10.1016/j.jccase.2020.07.018]

13. Yang $\mathrm{T}$, Hui $\mathrm{R}, \mathrm{Wu} \mathrm{Q}$, Tian J, Chen $\mathrm{H}$. An invasive thymoma extending into the superior vena cava and right atrium. Ann Transl Med 2019; 7: 498 [PMID: 31700934 PMCID: PMC1411540 DOI: 10.21037/atm.2019.08.59]

14. Lim KE, Shen TC. Surgical treatment of an invasive thymoma with intracaval and intracardiac extension. Acta Cardiol Sin 2017; 33: 204-206 [PMID: 28344425 PMCID: PMC5364163 DOI: 10.6515/ACS20160426A]

15. Afzal A, Wong I, Korniyenko A, Ivanov A, Worku B, Gulkarov I. Superior vena cava syndrome from an invasive thymoma with transcaval invasion to the right atrium. J Surg Case Rep 2016; 2016: rjw044 
[PMID: 27099229 PMCID: PMC4837328 DOI: 10.1093/jscr/rjw044]

16. De Giacomo T, Patella M, Mazzesi G, Venuta F. Successful resection of thymoma directly invading the right atrium under cardiopulmonary bypass. Eur J Cardiothorac Surg 2014; 48: 332-333 [PMID: 25293404 DOI: 10.1093/ejcts/ezu376]

17. Dong Y-Q, Liang J-S, Zhang X-M, Zhu S-B, Xu J-H, Ji T, Yin G-L. Surgical treatment of an invasive thymoma extending into the superior vena cava and right atrium. World J Surg Oncol 2014; $12: 6$ [PMID: 24400724 PMCID: PMC3938044 DOI: 10.1186/1477-7819-12-6]

18. Rizzardi G, Arena V, Passera E, Bortolotti L. Radical surgery in an unusual case of thymoma with intraluminal growth into the superior vena cava and right atrium. Interact Cardiovasc Thorac Surg 2013; 17: 1054-1055 [PMID: 23996733 PMCID: PMC2002361 DOI: 10.1093/icvts/ivt397]

19. Weis C-A, Yao X, Deng Y, Detterbeck FC, Marino M, Nicholson AG, Huang J, Ströbel P, Antonicelli A, Marx A, Contributors to the IRD. The impact of thymoma histotype on prognosis in a worldwide database. J Thorac Oncol 2015; 10: 367-372 [PMID: 25616178 PMCID: PMC1411540 DOI: 10.1097/JTO.0000000000000393]

\section{Figures}

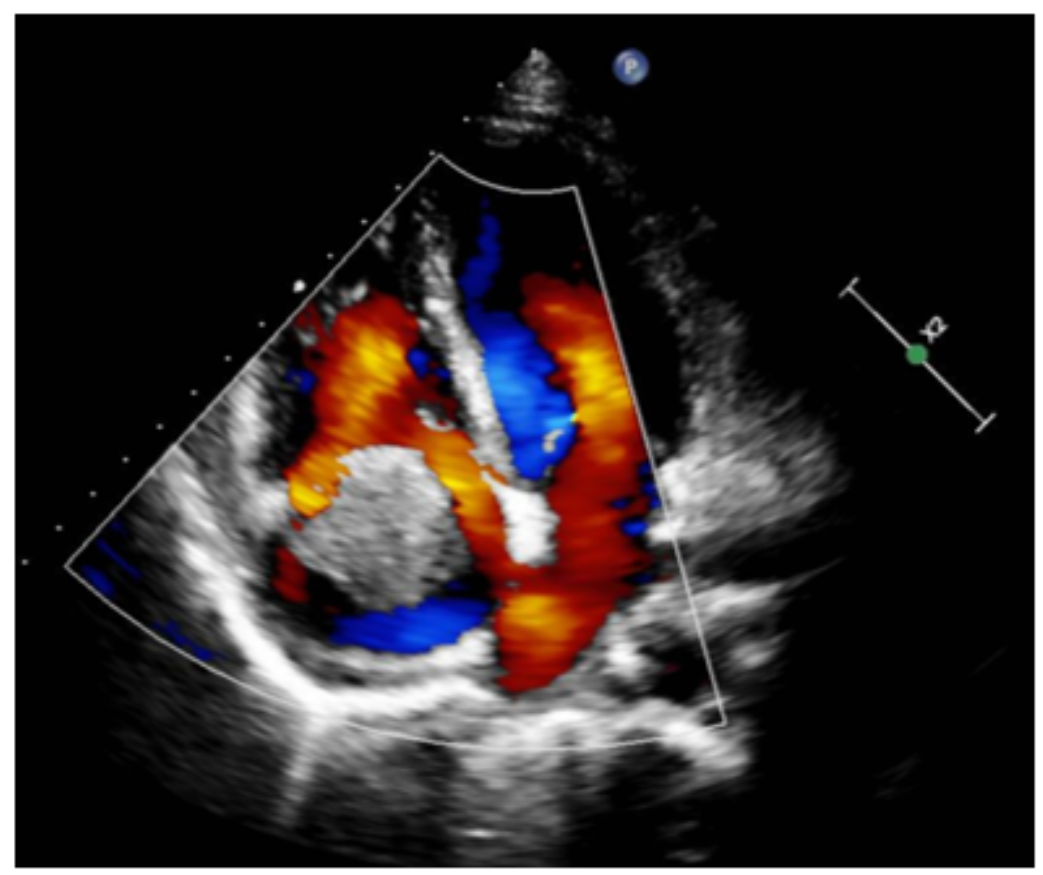

Figure 1

\section{Echocardiogram.}



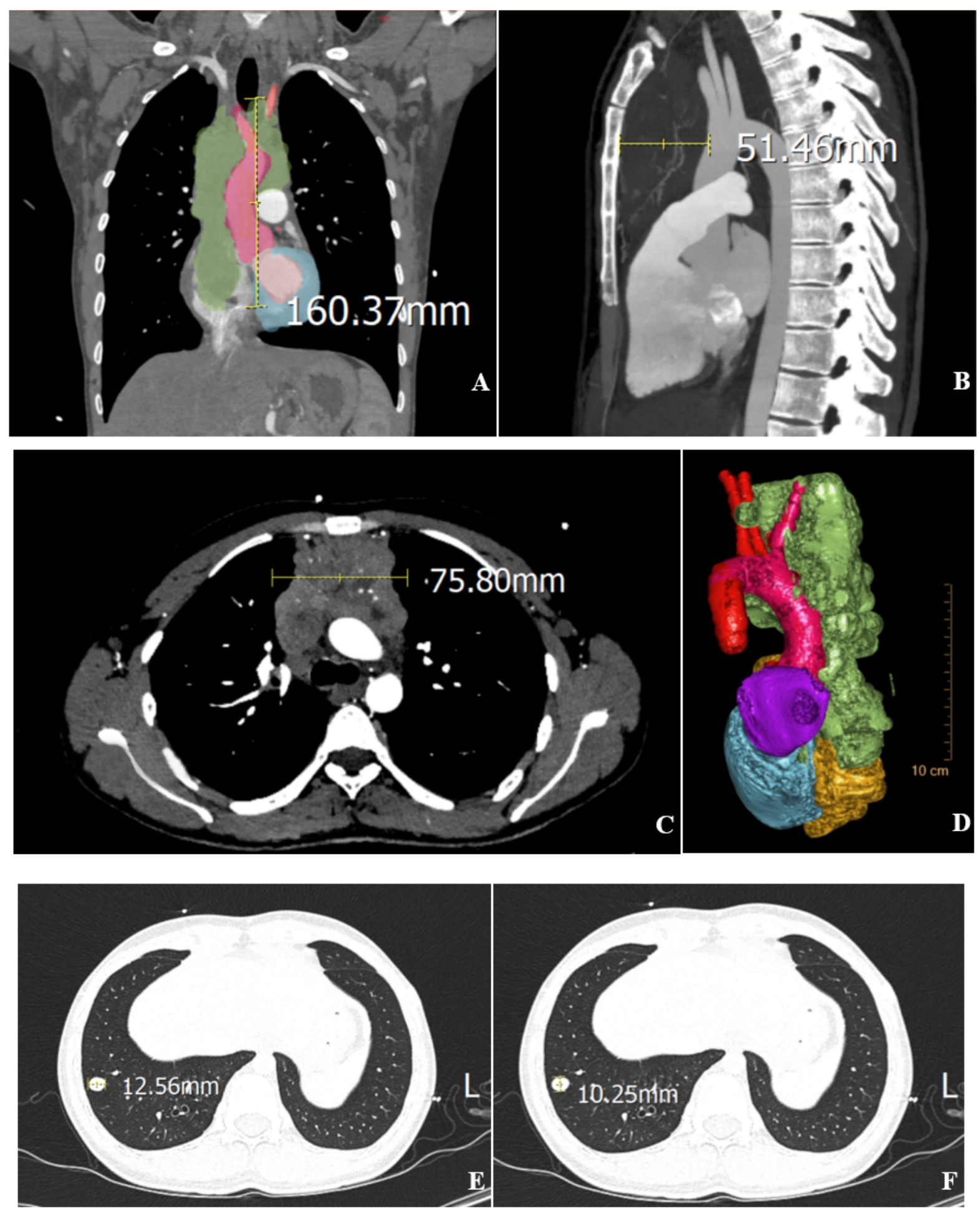

Figure 2

Enhanced CT scan with 3D reconstruction (tumor size of $16.0 \times 7.6 \times 5.2 \mathrm{~cm}$ ). A: Coronal view. Green, red, and blue areas are the tumor, aorta, and a portion of the left ventricular myocardium, respectively; $\mathbf{B}$ : sagittal view; C: cross-sectional view; D: virtual reality reconstruction; E and F: lung metastases (12.56 $\mathrm{mm} \times 10.25 \mathrm{~mm})$. 

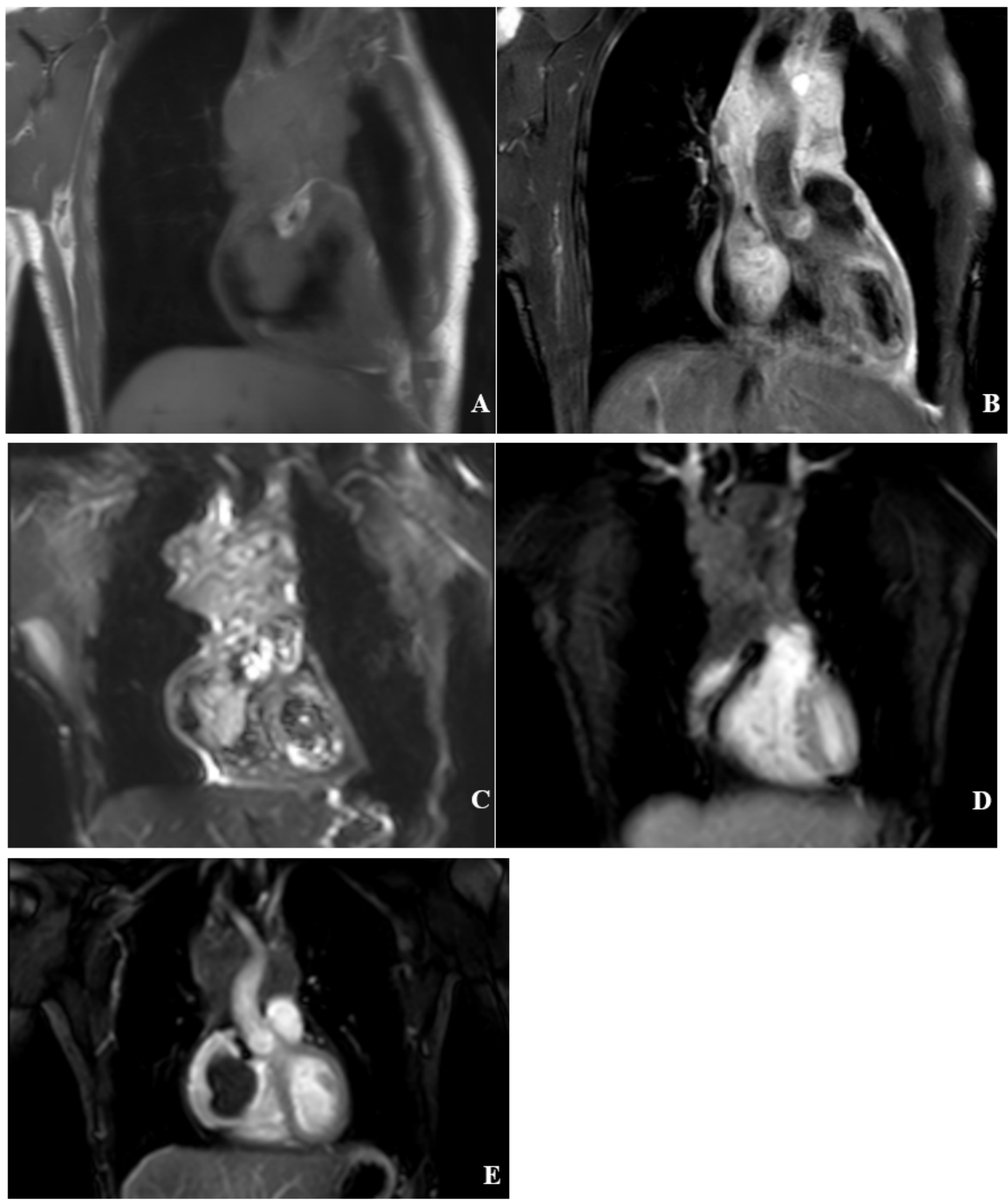

Figure 3

MRI cardiac and mediastinal scan with enhancement and multi-phase perfusion scan. A: T1WI shows slightly mixed signals; B: T2WI shows slightly mixed intense signals; C: DWI shows diffusion-limited high signal shadows; D and E: intense signal shadows are observed in the enhanced images. 

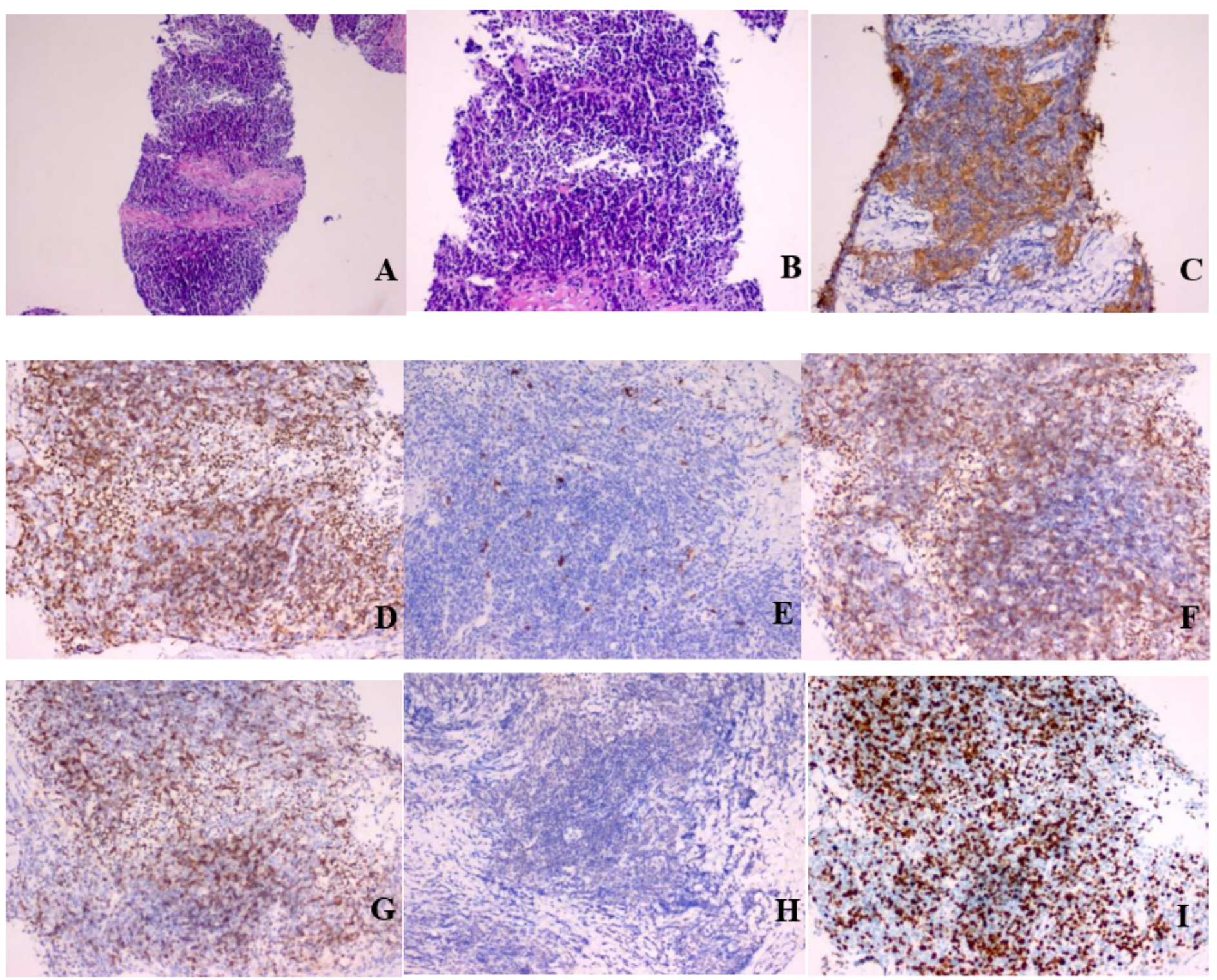

\section{Figure 4}

Pathological and immunohistochemistry examinations. A: Hematoxylin and eosin (H\&E) staining (100x); B: H\&E staining (200x); C: CK19 (200x); D: CD3 (200x); E: CD20 (200x); F: CD5 (200x); G: CD1a (200x); H: CD117 (200x); I: Ki67 (200x). 